\title{
An Upper Bound on the Entropy of Constrained 2d Fields
}

\author{
Forchhammer, Søren; Justesen, Jørn
}

Published in:

Information Theory, 1998. Proceedings. 1998 IEEE International Symposium on

Link to article, DOI:

10.1109/ISIT.1998.708656

Publication date:

1998

Document Version

Publisher's PDF, also known as Version of record

Link back to DTU Orbit

Citation (APA):

Forchhammer, S., \& Justesen, J. (1998). An Upper Bound on the Entropy of Constrained 2d Fields. In Information Theory, 1998. Proceedings. 1998 IEEE International Symposium on IEEE.

https://doi.org/10.1109/ISiT.1998.708656

\section{General rights}

Copyright and moral rights for the publications made accessible in the public portal are retained by the authors and/or other copyright owners and it is a condition of accessing publications that users recognise and abide by the legal requirements associated with these rights.

- Users may download and print one copy of any publication from the public portal for the purpose of private study or research.

- You may not further distribute the material or use it for any profit-making activity or commercial gain

- You may freely distribute the URL identifying the publication in the public portal

If you believe that this document breaches copyright please contact us providing details, and we will remove access to the work immediately and investigate your claim. 


\title{
An Upper Bound on the Entropy of Constrained 2d Fields
}

\author{
Søren Forchhammer and Jørn Justesen \\ Dept. of Telecommunication, 371 \\ Technical University of Denmark \\ DK-2800 Lyngby, Denmark \\ Email sf@tele.dtu.dk, jju@tele.dtu.dk
}

\begin{abstract}
An upper bound on the entropy of constrained $2 \mathrm{~d}$ fields is presented. The constraints have to be symmetric in (at least) one of the two directions. The bound generalizes (in a weaker form) the bound of Calkin and Wilf which is valid only for processes with symmetric transfer matrices. Results are given for constraints specified by run-length limits and minimum distance between pixels of the same color.
\end{abstract}

\section{INTRODUCTION}

We consider the (maximum) entropy of two-dimensional (2d) fields defined by a set of constraints. A well-known example (the hard square) is a binary field for which the maximum run-length of one of the colors is one in both directions. We consider shift invariant constraints of finite extent $(N, M)$, where each element is taken from an alphabet $A$ of size $|A|$. The $|A|^{N M}$ possible configurations on the $N$ by $M$ rectangle are divided in two sets of admissable and non-admissable configurations, respectively. Let $F(n, m)$ be the number of admissable configurations on an $n$ by $m$ rectangle, not violating the constraints within the rectangle. The per symbol entropy, $H$ may be defined as:

$$
H=\lim _{n, m} \frac{\log F(n, m)}{n m} .
$$

For bands of finite width $m$ and arbitrary height, a 1d approach may be applied. The states are given by $m$ by $N-1$ elements, so the states of a transition covers the necessary $N$ rows specified by the constraint. The entropy, $H(m)$, of the $m$ elements of a band of width $m$ may be determined as $l o g$ of the largest eigenvalue $\lambda_{\max }$ of the tranfer matrix, $\mathrm{T}$ of the $1 \mathrm{~d}$ process.

\section{UPPER BOUND ON ENTROPY}

For all positive integers $p$, the largest eigenvalue, $\lambda_{\max }$ of any real symmetric matrix satisfies

$$
\lambda_{\max } \leq \operatorname{Trace}\left(\mathbf{T}^{2 p}\right)^{1 / 2 p} .
$$

Transfer matrices, $\mathbf{T}$ are real and thus the only additional requirement is that they are symmetric. Calkin and Wilf [1] made the observation that the trace of $\mathbf{T}^{2 p}$ is composed of the states which map onto themselves after $2 p$ transitions. All of these solutions may form a cylinder of circumference $2 p$ and the trace gives the number of distinct solutions on this cylinder. This applies for any cylinder height, $n$. Taking the logarithm and making the cylinders infinitely high $(n \rightarrow$ $\infty$ ), Calkin and Wilf proved that the entropy of the cylinder process, $H^{\prime}(2 p)$ is an upper bound on the entropy of the hard square problem. For higher order processes the transfer matrix is not symmetric in general even if the process is symmetric. In this case, we shall specify a less restrictive process based on a modified cylinder. A two seam cylinder, is composed of two bands of width $p+M-1$ each satisfying the constraints. To control the constraint, an overlap of $M-1$ columns at each side is used. Instead of combining the two bands with an overlap of $M-1$ at each edge to form an ordinary cylinder, the state $i$ of e.g. the leftmost $M-1$ columns are mirrored i.e. taken in reverse order before performing the overlap. In both cases we end up with $2 p$ columns in the cylinder. The entropy of the two seam cylinder is denoted $H^{\prime \prime}(2 p)$.

Theorem 1 For processes specified by shift invariant constraints of finite extent $(N, M)$, and symmetric in (at least) one direction, the entropy is upper bounded as

$$
H \leq \max \left\{\frac{H^{\prime}(2 p)}{2 p}, \frac{H^{\prime \prime}(2 p)}{2 p}\right\}
$$

where $p$ is a positive integer and $2 p \geq 2 M-2$.

The proof is based on the construction of a process where the two types of cylinders generate the admissable configurations as a subset of a process with symmetric transfer matrix.

\section{RESULTS}

In the experiments reported below, $H^{\prime \prime}(2 p)>H^{\prime}(2 p)$, which seems reasonable to conjecture (under some prerequisites). The results are for binary fields.

Constraint 1: Minimum distance (1-norm) of 3 between $f$ 's. The best upper bound on entropy by (3) is $H<0.3569=$ $H^{\prime \prime}(14) / 14\left(H^{\prime}(14) / 14=0.3503\right)$, improving the previous best upper bound $H<H(14) / 14=0.3597$ [2]. The best lower bound in [2] is $H>0.350306$. It may be remarked that the cylinder entropy $H^{\prime}(10)$ is below this lower bound and thus can not be an upper bound. The estimated entropy is $H \approx$ $H(16)-H(15)=0.35030719$ [2].

Constraint 2: Minimum distance ( $\infty$-norm) of 3 between 1 's. The best upper bound on the entropy by (3) is $H<$ $0.2432=H^{\prime \prime}(14) / 14 .\left(H^{\prime}(14 / 14=0.2379)\right.$. $)$ In [3], $0.25681>$ $H>0.22257$ and the estimate, $H \approx 0.235$ are reported.

Constraint 3: Maximum run-length of 2 for both values and in both directions. The best upper bound by (3) is $H<0.4728=H^{\prime \prime}(10) / 10\left(H^{\prime}(10) / 10=0.4650\right)$, improving the upper bound using bands, $H<H(10)=0.5079$. $H>0.4650$ and $H \approx H(10)-H(9)=0.4682$ were reported in [2].

\section{REFERENCES}

[1] N. J. Calkin and H. S. Wilf, "The number of independent sets in a grid graph," SIAM Journal of Discrete Mathematics, vol. 11, pp. 54-60, 1998.

[2] S. Forchhammer and J. Justesen, "Entropy bounds for constrained two-dimensional random fields," IEEE Trans. Inform. Theory to appear.

[3] W. Weeks IV, Checkerboard codes, Master Thesis, University of Illinois, Urbana-Champaign, 1996. 